\title{
ANÁLISIS MORFOMÉTRICO E HIDROCLIMATOLÓGICA EN LA ZONA DEL PARQUE NATURAL AGUAS DE RAMÓN
}

\author{
Valentina Vergara \\ Universidad Tecnológica Metropolitana, Ingeniero Geomensor por el Departamento de \\ Industria, Santiago, Chile \\ valentina.vergara23@gmail.com
}

Pablo Ulloa

Universidad Tecnológica Metropolitana, Ingeniero Geomensor por el Departamento de Industria, Santiago, Chile

pablo.ulloam@utem.cl

\section{Miguel Moraga}

Universidad Tecnológica Metropolitana, Ingeniero Geomensor por el Departamento de Industria, Santiago, Chile migue.moraga7@gmail.com

Keyla Manuela Alencar da Silva Alves

Universidad Tecnológica Metropolitana, Profesora Asistente del Departamento de Industria,

Santiago, Chile

Keyla.dasilva@utem.cl

\begin{abstract}
RESUMEN - En base a los registros meteorológicos y modelos digitales de terreno obtenidos de la Quebrada de San Ramón, se realizó una caracterización hidroclimatológica y morfométrica de la zona, con aportes de climogramas para análisis de cantidad de precipitaciones y temperaturas en ciertos periodos de tiempo. En la metodología se realizaron los cálculos de redes de drenaje y cálculos de parámetros morfométricos, estimación del tiempo de concentración (aproximadamente de 36.87 minutos) y velocidad media del flujo (aproximadamente de $15.1 \mathrm{~km} / \mathrm{h}$ ). Las respuestas hidrológicas de la quebrada estudiada están determinadas principalmente por la topografía de la zona, pendientes, relieves, tipo de suelo, cantidades de agua caída y direcciones del flujo de agua. El acotado tiempo de concentración y la velocidad de flujo de agua lenta, en conjunto al curso de agua, pendiente y magnitudes extremas de precipitación se traducen en una zona de peligrosidad debido a la susceptibilidad de flujos rápidos capaces de transportar altas cargas de sedimento detrítico.
\end{abstract}

Palabras-chave: Geomorfologia, Hidrologia, Hidroclimatología, Jerarquía de drenaje.

\section{ANÁLISE MORFOMÉTRICA E HIDROCLIMATOLÓGICA NA ÁREA DO PARQUE NATURAL AGUAS DE RAMÓN}

RESUMO - Com base nos registros meteorológicos e nos modelos digitais de terrenos obtidos a partir da Quebrada de San Ramón, foi realizada uma caracterização hidroclimatológica e morfométrica da área, com contribuições de climas para análise da quantidade de chuvas e temperaturas em determinados períodos de tempo. Na metodologia, foram realizados cálculos de redes de drenagem e cálculos de parâmetros morfométricos, estimativa do tempo de concentração (aproximadamente 36,87 minutos) e velocidade média do fluxo (aproximadamente 15,1 km / h). As respostas hidrológicas da ravina estudada são determinadas principalmente pela topografia da área, declives, relevos, tipo de solo, quantidades de água caída e direções do fluxo da água. O tempo limitado de concentração e a lenta taxa de fluxo de água, juntamente com o curso da água, a 
inclinação e as magnitudes extremas de precipitação, se traduzem em uma zona de perigo devido à suscetibilidade de fluxos rápidos capazes de transportar altas cargas de sedimentos detríticos.

Palavras-chave: Geomorfologia, Hidrologia, Hidroclimatologia, Hierarquia de Drenagem.

\title{
MORPHOMETRIC AND HYDROCLIMATOLOGICAL ANALYSIS IN THE AREA OF THE NATURAL PARK AGUAS DE RAMÓN
}

\begin{abstract}
Based on the meteorological records and digital terrain models obtained from the Quebrada de San Ramón, a hydroclimatological and morphometric characterization of the area was carried out, with contributions from climograms to analyze the amount of rainfall and temperatures in certain periods of time. In the methodology, drainage network calculations and morphometric parameter calculations, estimation of the concentration time (approximately 36.87 minutes) and average flow velocity (approximately $15.1 \mathrm{~km} / \mathrm{h}$ ) were performed. The hydrological responses of the stream studied are determined mainly by the topography of the area, slopes, reliefs, type of soil, amounts of water fall and directions of water flow. The limited concentration time and the slow water flow velocity, together with the water course, slope and extreme amounts of precipitation, result in a danger zone due to the susceptibility of fast flows capable of transporting high loads of detrital sediment.
\end{abstract}

Keywords: Geomorphology, Hydrology, Hydroclimatology, Drainage hierarchy.

\section{INTRODUCCIÓN}

Durante las últimas décadas se han registrado una serie de eventos aluviales y de crecidas y caudales importantes que han afectado de manera significativa a la precordillera de la región metropolitana de Santiago, lo que ha representado un aumento en el peligro en especial por el aumento de urbanización en las cercanías de la cordillera. Entre los hechos ocurridos, los más significativos fueron en los años 1983, 1986, 1993, 2005, 2008, 2016, en lo cual el de mayor magnitud de impactos ocurrido el 3 de mayo de 1993 el cual dejó decenas de víctimas y grandes cantidades de pérdidas económicas (CONTRERAS, 2016; VARGAS, 1999). El dicho evento hidrometeorológico corto e intenso del 1993, con características cálidas, asociado a los efectos del fenómeno del niño, desencadenaron un importante escurrimiento de agua y barro principalmente en las cuencas de la Quebrada de Macul y la Quebrada de Ramón, siendo ésta última en donde se enfoca este trabajo, sector que se convirtió en un Parque Ecológico Educativo, denominado Parque Natural Aguas de Ramón, para la conservación y protección de la Cordillera de Santiago de Chile (LARA, 2009, ACUÑA et.al., 1995).

Este estudio buscó caracterizar la morfometría de la microcuenca e hidroclimatológica donde se ubica el Parque Natural Aguas de Ramón. Para esto fue realizado una visita al sitio en el día 12 de mayo 2018, en donde se registraron varios puntos de controle con la ayuda de una aplicación llamada Kobo Toolbox, la cual cuenta con la opción de guardar puntos geográficos, una pequeña descripción y sus coordenadas con el GPS integrado al celular, con lo cual se obtuvo la ruta recorrida y los lugares donde se apreciaron cicatrices de deslizamientos, caídas de rocas, depósitos de talus y otros tipos de remociones en masas. Por ser una zona de alta pendiente, es común identificar sectores donde se ha producido remociones, sean estas gatilladas por factores climáticos o gravitacionales. La ruta tomada en el parque fue la de $6.5 \mathrm{~km}$ llamada "Los Peumos", es un trekking básico y altamente señalizado.

La caracterización climática del área de estudio fue complementada con información recopilada de trabajos realizados en esta misma zona y datos climatológicos obtenidos desde el Servicio Nacional de Geología y Minería (SERNAGEOMIN, 2015), donde se obtuve informaciones relacionadas a la precipitación, temperatura, humedad, velocidad del viento, etc.

Una vez seleccionados los datos y aplicadas las metodologías, se pudo caracterizar y analizar los aspectos morfométricos, hidrológicos y climatológicos de la microcuenca. Tales resultados contribuyen con la identificación de posibles puntos críticos de flujos rápidos, en donde podrían ser reactivados provocando remociones en masas debido a la ocurrencia de eventos hidroclimáticos 
severos. Lo que significaría una amenaza a la población que ocupa las áreas cercas a la microcuenca. Este estudio pretende generar información básica de carácter geomorfológico e hidrológico que pueda contribuir con estudios de planificación de riesgo futuro, monitoreo de respuesta temprana para situaciones específicas, como lluvias concentradas o crecidas de caudal, con el fin de disminuir el riesgo para la población (RUGIERRO y WYNDHAM, 2013).

\section{ÁREA DE ESTUDIO}

La microcuenca hidrográfica Quebrada San Ramón se ubica en la zona oriente de Santiago, en las comunas de La Reina y Las Condes. tiene su inicio en el cordón del Cerro San Ramón (3253 m.s.n.m.), al este de Santiago, y desciende hacia el valle con un sentido general N-S en su tramo superior y E-W en su tramo inferior y está definido por las coordenadas angulares $33^{\circ} 26^{\prime} 00^{\prime \prime} \mathrm{de}$ latitud sur y $70^{\circ} 31^{\prime} 00^{\prime \prime}$ de longitud oeste (Figura 1).

Figura 1. Localización de la microcuenca donde se ubica el Parque Natural Agua de Ramon

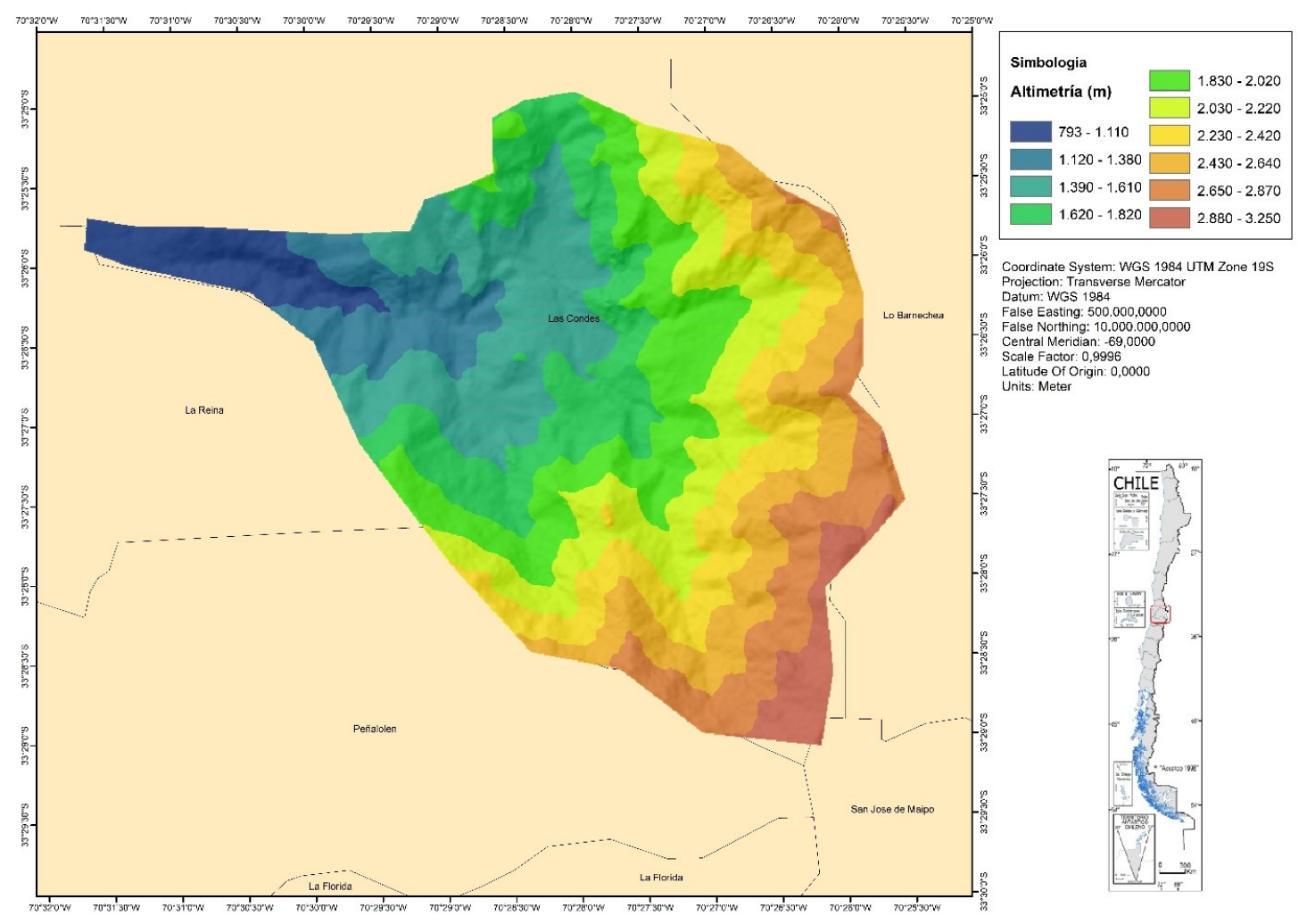

Fuente: Elaborado por los autores

La litología de la zona está conformada por unidades, tanto antiguas como recientes, que cubren la roca base, que corresponde principalmente a brechas, lavas y tobas otorgadas a la Formación Abanico. Más específicamente en la parte alta de esta hay rocas asignadas a la formación de abanicos, que corresponden a rocas volcánicas con algunas intercalaciones sedimentarias de areniscas y lutitas. Estas rocas son instruidas por cuerpos de basaltos, granitos y granodioritas de tamaño variable. Estas rocas son afectadas a distintas escalas por diaclasas, pliegues y zonas de falla. En cambio, la parte baja de la quebrada corresponde al abanico aluvial de la misma y consiste en una serie de depósitos fluviales no consolidados, compuestos principalmente por gravas, con niveles de arenas gruesas a medias y por depósitos de flujos de detritos o barro intercalados de granulometría muy heterogénea (IBÁÑES, MORENO y GISBERT, 2011). 
La gran mayoría de estos materiales se encuentran desprendido, en laderas o cauces, siendo susceptibles a ser movilizados como flujos o deslizamientos. Los procesos aluviales y de remoción en masa, principalmente flujos de detritos han generado un abanico aluvial de gran extensión, que cubre parte de la zona urbana de la comuna de La Reina (NARANJO y VARELA, 1996).

El área de la microcuenca alcanza sus máximas alturas en las cumbres del cerro San Ramón con 3253 m.s.n.m., hasta los alrededores de la cota 2000 m.s.n.m., la quebrada de San Ramón presenta pendientes fuertes en promedio de $20^{\circ}$ a $30^{\circ}$, desarrollando algunos saltos y rápidos de agua, en los tramos que están entre los 1650 m.s.n.m. y los 1450 m.s.n.m. la pendiente es suave (menor a $\operatorname{los} 10^{\circ}$ ).

Esta microcuenca presenta características geomorfológicas y geológicas propicias para el movimiento de masas y fue fuente de uno de los mayores eventos de flujos de detritos ocurridos en la región en el último tiempo, en el año 1993. La cercanía con la ciudad y la constante presión de crecimiento urbano sobre los faldeos cordilleranos hacen que en el lugar estos fenómenos constituyan una amenaza importante. Este crecimiento en la actualidad afecta sectores que naturalmente son zonas de deposición de material transportado durante eventos de remociones en masa que han ocurrido en la montaña (RÍOS BRIONES, 2015).

\section{METODOLOGÍA}

En este estudio tubo inicio con el trabajo de terreno en una parte delimitada de la microcuenca que se encuentra en el Parque Natural Aguas de Ramon, allí se observó y registraron los puntos de control donde se localizaban cicatrices de zona de arrastre. Para el registro de los puntos se utilizó la aplicación android KoBo Collect v1.14.0 para la recolección de datos en terreno, dado que permite realizar una encuesta para realizar un mapeo colectivo, tomando datos GPS y agregando descripciones del punto y una foto si fuese necesario; luego estos datos fueron descargados en formato KMZ, y se cargaron en Google Earth para una transformación de formato a KML (Figura 2), para posteriormente poder ser cargados al software ArcMap en conjunto con la imagen satelital obtenida desde el satélite ALOS.

Figura 2. Puntos de control de las cicatrices de zonas de arrastre 


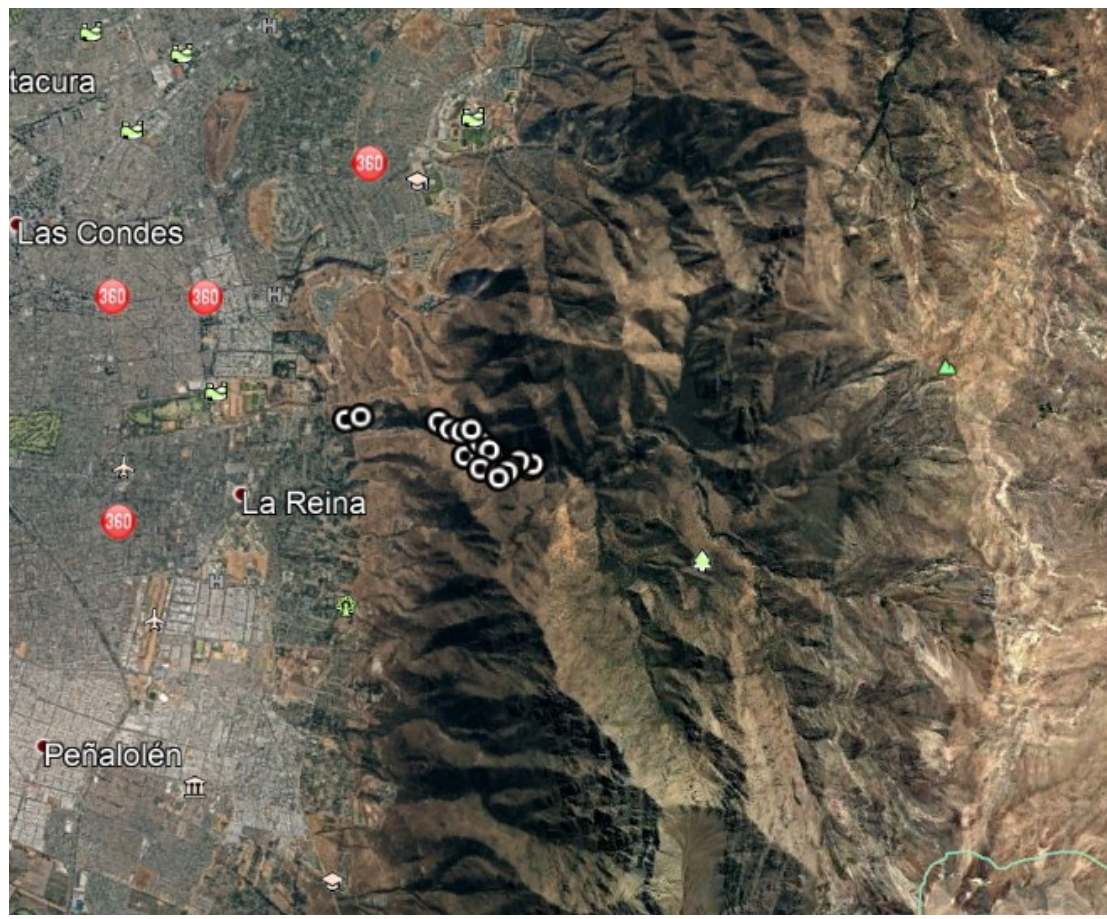

Fuente: Elaborado por los autores

A la etapa siguiente al trabajo en terreno se realizó la caracterización de la red de drenaje de la zona a partir de la ejecución de cálculos usando las herramientas de SIG's que identifican la dirección y zonas de acumulacion de los flujos. La red de drenaje fue identificada a partir de la clasificación de los ríos en Chile, el cual es 150 metros de buffer, donde se clasificó los cauces y arroyos a partir de las fórmulas de Strahler. Está metodología arrojó una red de drenaje donde se realiza un orden en los cauces a partir de parámetros de jerarquía, el área, perímetro, largo y ancho de la microcuenca.

El ordenamiento y la jerarquización de la red de drenaje se ejecutaron con base en los criterios de Horton (1945) modificados por Strahler (1964), se elaboraron perfiles topográficos longitudinales del cauce principal de río estudiado y a la vez se realizaron las mediciones y cálculos morfométricos básicos para la microcuenca con la plataforma ArcMap y con ayuda del software Microsoft Excel según las ecuaciones matemáticas que definen los parámetros a estudiar.

El tiempo de concentración (Tc) de la cuenca en la salida del río estudiado se estimó según la ecuación dada por California Culvert Practice:

$$
T c=\left[0.87075 * L^{3} / H\right]^{0.385}
$$

Donde Tc es el tiempo de concentración en horas, para obtenerlo en minutos se debe multiplicar por 60, L es la longitud de la cuenca $(\mathrm{km})$, y H es la diferencia entre la altura máxima y mínima $(\mathrm{m})$. La velocidad media del flujo en el cauce principal se calculó en la misma sección hidráulica de interés, por medio de la ecuación

$$
\mathrm{Vmf}=\mathrm{L} / \mathrm{Tc}
$$

Vmf es la velocidad media del flujo $(\mathrm{km} / \mathrm{h})$, $\mathrm{L}$ es la longitud del cauce principal $(\mathrm{Km})$ y Tc es el tiempo de concentración anteriormente obtenido.

Se caracterizó el régimen pluviométrico a través de precipitaciones y temperaturas medias obtenidas de la Dirección General de Aguas del Ministerio de obras públicas, donde se pudo obtener datos históricos de una estación meteorológica cercana al sitio estudiado a partir del año 2014 hasta la actualidad. Con esta información se realizaron climogramas del área estudiada, encontrándose en su eje horizontal las fechas de los datos, el eje vertical principal las precipitaciones y el eje vertical secundario las temperaturas, presentado en forma de columnas y líneas respectivamente. Gracias a este gráfico se pueden realizar análisis de la cantidad de agua caída y las temperaturas que existen en 
esta área en los diferentes periodos mensuales a través de los años. También se realizó de la misma manera un climograma anual con los mismos datos anteriores para poder observar de manera más amplia las diferencias de precipitación, temperaturas en periodos más amplios (GIRONÁS y SANDOVAL, 2016; MÉNDEZ et. al., 2015)

\section{RESULTADOS}

\section{Morfometría de la microcuenca y la red de drenaje}

Las magnitudes calculadas para los parámetros estudiados corresponden a la variable de escala que posee la microcuenca, cuya apreciación depende notoriamente de la escala de la cartografía utilizada para las mediciones. El conjunto de magnitudes y parámetros manifiesta un sistema hidrogeomorfológico de pequeñas dimensiones, con que ayuda con la clasificación de la cuenca como una microcuenca.

El área que se ha estudiado, por ser pequeña dentro de los patrones de cuenca, por sí sola depende de menores tiempo de concentración de la escorrentía superficial y mayor susceptibilidad de experimentar crecidas con hidrogramas de alturas pronunciadas y de breve duración.

Este valor areal también manifiesta que la superficie es una fuente que no tiene un real aporte a la escorrentía superficial, y que, al presenciar una tormenta de magnitud considerable o extrema, toda la superficie se activa y aporta agua, lo que ayuda a la creación de hidrogramas disparados y de cortas duraciones.

Por otro lado, el valor de área, los valores de ancho y longitud de la microcuenca sugieren un sistema de drenaje con redes que tiende a crecer de forma longitudinal, lo que se interpreta como una respuesta al curso de agua que drenan en el sentido de la pendiente presente en el terreno, esto condicionado por la poca resistencia a los procesos erosivos. Los valores de diámetro y perímetro también se relacionan a las pequeñas dimensiones de la microcuenca.

En la zona estudiada, las alturas están comprendidas entre $793 \mathrm{msnm}$ y $3254 \mathrm{msnm}$, las diferencias entre estas dos alturas definen un relieve máximo de $2461 \mathrm{msnm}$ y un radio de relieve de $265.2525 \mathrm{msnm} / \mathrm{km}$ lo que denota una altitud y pendiente propicia para la aparición de procesos erosivos y transporte de materiales.

El valor obtenido de la pendiente media de la cuenca es alto, lo que va de la mano con los resultados de alturas y es de esperarse al estudiar un sector montañoso, pero aun así se demuestra la diferencia entre la parte baja de la microcuenca, y la alta, siendo la alta en donde se observan mayores procesos erosivos mientras que la baja es una área depositacional (abanico aluvial), la presidencia de mayores alturas condicionan tiempos menores de concentración, mayor potencial erosivo y arrastre de sedimentos.

La obtención de los perfiles transversales lo largo del cauce principal denota una profundidad leve, lo que propicia la inundación cuando existan tormentas de grandes magnitudes, entendiendo que la velocidad media de flujo es pequeña. Los perfiles transversales tienen una diferencia de altura de unos 200 metros aproximadamente, lo que apoya la teoría de la factibilidad de inundación en la zona baja del cauce principal.

Tabla 1. Parámetros morfométricos medidos y calculados de la cuenca y de la red de drenaje

\begin{tabular}{lll}
\hline Parámetro & unidades & magnitud \\
\hline Área de la cuenca & $\mathrm{km} 2$ & 359.310 \\
\hline Área rectángulo equivalente & $\mathrm{km} 2$ & 359.310 \\
\hline Área vertiente mayor & $\mathrm{km} 2$ & 0.7998 \\
\hline
\end{tabular}




\begin{tabular}{lll}
\hline Área vertiente menor & $k m 2$ & 143.030 \\
\hline Perímetro & $k m$ & 282.725 \\
\hline Longitud & $k m$ & 92.780 \\
\hline Ancho medio & $k m$ & 38.727 \\
\hline Diámetro & $k m$ & 67.638 \\
\hline Perímetro de un círculo igual a la cuenca & $k m$ & 212.490 \\
\hline Relieve máximo & $\mathrm{msnm}$ & 3254 \\
\hline Radio del relieve & $\mathrm{msnm} / \mathrm{km}$ & 2.652 .526 \\
\hline Pendiente media & $\mathrm{m} / \mathrm{m}$ & 273.937 \\
\hline Pendiente media & $\% / \%$ & 544.923 \\
\hline Largo del cauce principal & $\mathrm{km}$ & 24.751 \\
\hline Coeficiente de gravelius & - & 13.305 \\
\hline Elongación & - & 0.6461 \\
\hline Perímetro relativo crenulación & - & 222.464 \\
\hline Coeficiente de compacidad & - & 13.206 \\
\hline Índice de alargamiento & - & 23.957 \\
\hline Índice de homogeneidad & - & 1 \\
\hline Índice de simetría & - & 0.0559 \\
\hline Factor forma & - & 0.4174 \\
\hline Alargamiento caquot & - & 15.478 \\
\hline Radio elongación & - & 0.7290 \\
\hline Radio de circularidad & - & 0.5649 \\
\hline Índice de forma & - & 0.1252 \\
\hline Índice de largo y área & - & 0.1882 \\
\hline Coeficiente de forma & - & 0.4174 \\
\hline Coeficiente de redondez & - & 18.816 \\
\hline Tiempo de concentración & $\mathrm{horas}$ & 0.6145 \\
\hline Tiempo de concentración & $\mathrm{km} / \mathrm{h}$ & 368.719 \\
\hline Velocidad media del flujo & & 150.976 \\
\hline & & \\
\hline & & \\
\hline
\end{tabular}

Los perfiles transversales del cauce principal dan un sentido claro de la inundabilidad existente en base a la profundidad de cada tramo estudiado (Figura 3).

Figura 3. Perfiles transversales de la corriente principal del río

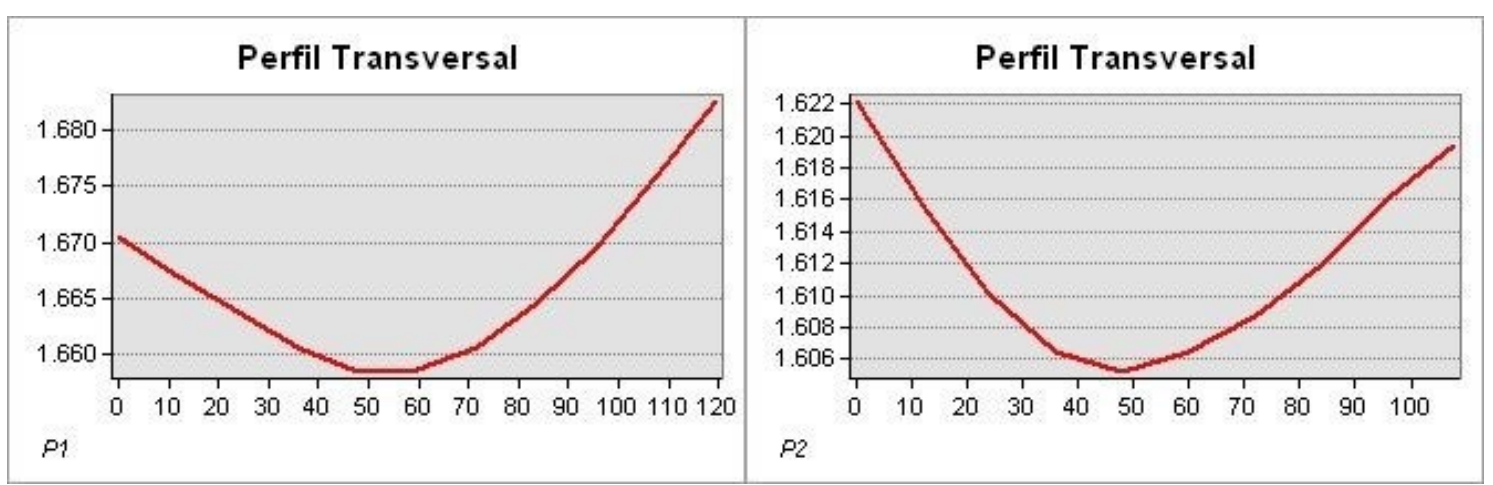



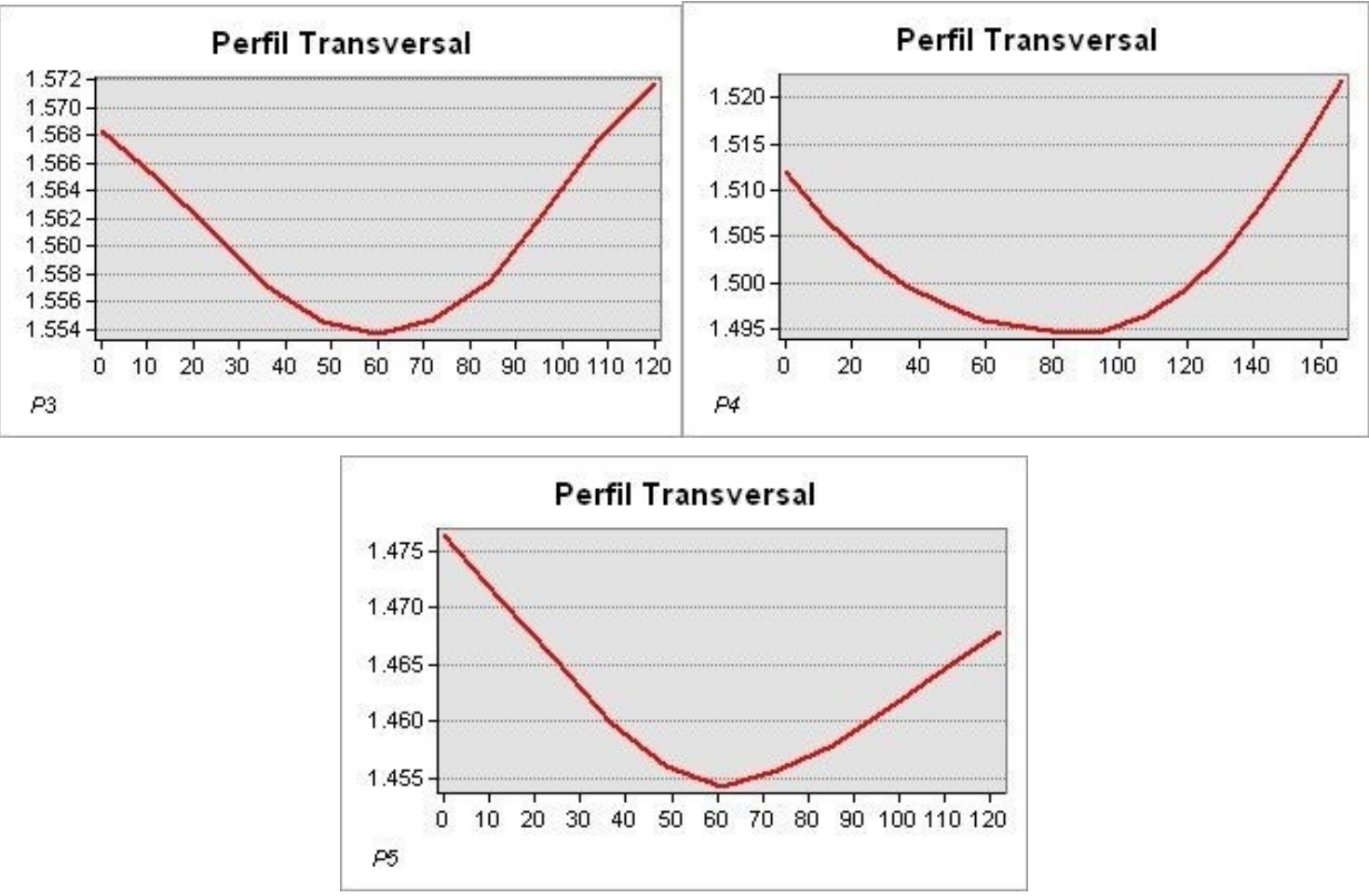

La expresión morfológica del trazado del perímetro de la cuenca sobre la base cartográfica en análisis evidencia la forma longitudinal y alargada. El valor de la elongación indica la forma alargada con una configuración rectangular, además este valor es representativo en cuencas emplazadas en relieves montañosos.

El valor del perímetro relativo de crenulación se condice con los valores anteriormente mencionados, ya que indican que tiene una tendencia a ser alargada pero no en gran manera, el índice de forma es bastante bajo lo que indica el alargamiento de la microcuenca y la baja posibilidad de crecidas, sin embargo tomando en cuenta la climatología de la región, donde se encuentran varias estaciones de altas temperaturas y una época invernal de corta duración y con eventos donde se disparan las precipitaciones, es que se entiende que la extensión de la tormenta, duración e intensidad de esta, siempre considerando que se estudia un sistema hidrogeomorfológico pequeño.

El coeficiente de compacidad define la forma como oval redonda a oval oblonga, indicando que es una forma cercana a la circularidad, por ende, las crecidas pueden ser más frecuentes, en donde el tiempo de concentración demuestra la capacidad de inundación en conjunto con su topografía y en presencia de una tormenta.

La existencia de sistemas de fracturas (diaclasas y fallas) y los desniveles topográficos entre bloques tectónicos ejercen control en la disposición y distribución de las corrientes que conforman la red de drenaje de la microcuenca.

El tiempo de concentración es de $36.8719 \mathrm{~min}$, con una velocidad media de flujo $15.0976 \mathrm{~km} / \mathrm{h}$ demuestra que las aseveraciones realizadas con anterioridad son verídicas, puesto que el tiempo de concentración es corto, y la velocidad es baja, por ende, en una crecida del caudal gracias a una tormenta de gran envergadura haría colapsar la cuenca, teniendo en cuenta que la velocidad es baja y no podría evacuar toda la cantidad de agua precipitada y el material erosionado sería transportado.

La caracterización climática de la zona fue realizada en base a los datos de precipitación anuales y mensuales, con objetivo de identificar y comprender la dinámica de las lluvias, y si en los últimos años las precipitaciones han presentado alguna tendencia positiva o negativa. A través del primer 
climograma (Figura 4) se puede observar una creciente en la magnitud de precipitaciones concentradas en los mismos meses en todos los años analizados, mientras que las temperaturas promedio se mantienen en su magnitud y en sus periodos mensuales. Este resultado demuestra que en los últimos cinco años los datos de precipitaciones no han demostrado tendencias significativas que representen un aumento o disminución de las lluvias. En el segundo se puede observar que en los años 2016 y 2017 hubo una crecida en la cantidad de precipitaciones, manteniendo una baja variación en sus temperaturas, pero eso no representa una tendencia de aumento (Figura 5).

Figura 4. Climograma mensual correspondiente al periodo de 2014 a 2018.

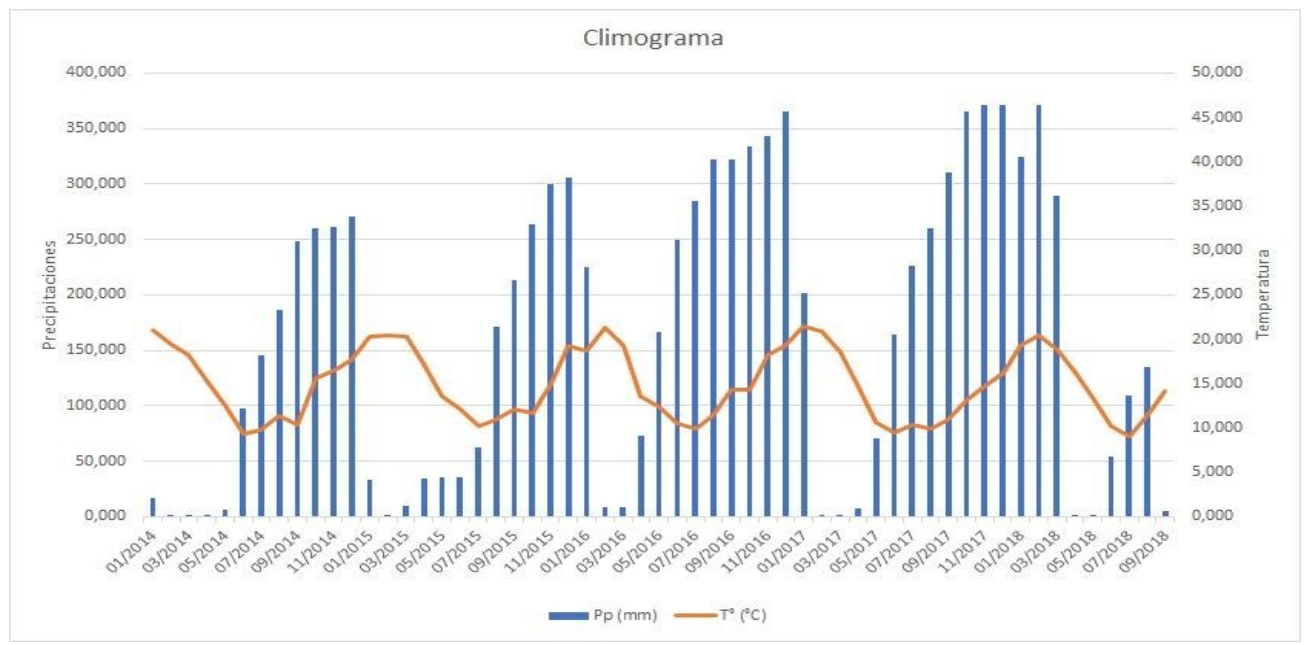

Fuente: elaboración de los autores

Figura 5. Climograma interanual correspondiente al periodo de 2014 a 2018.

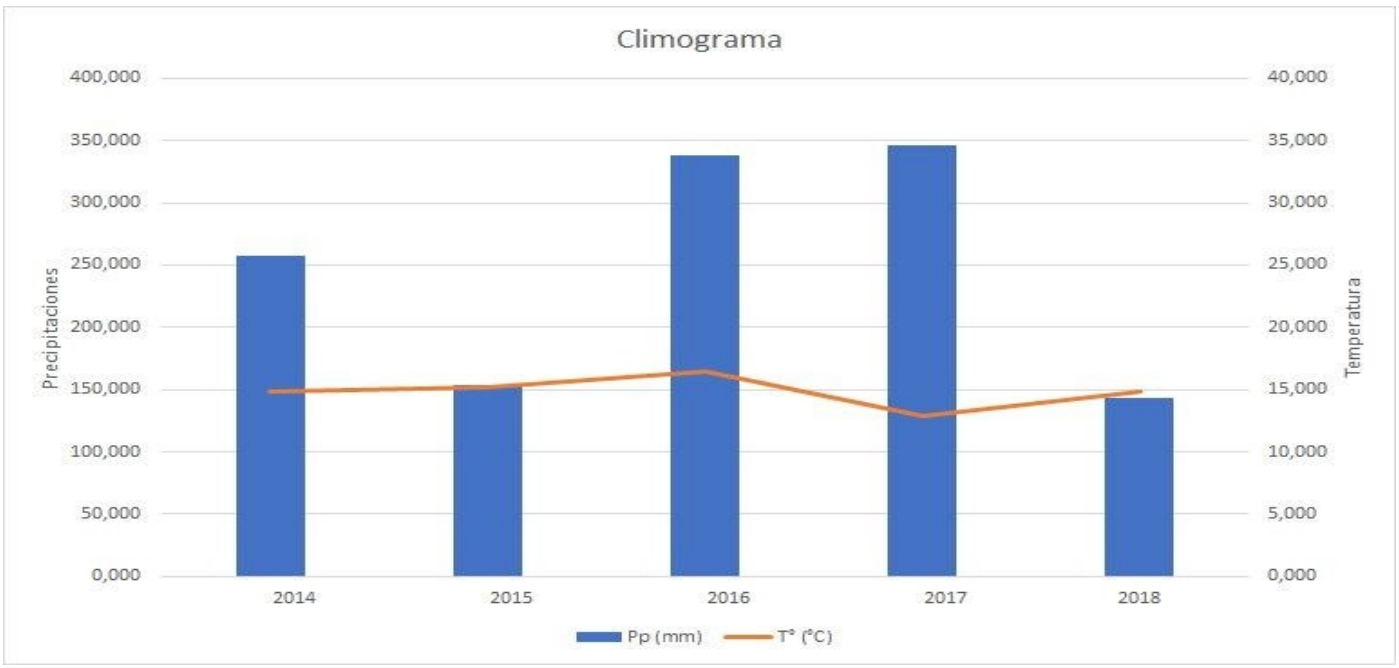

Fuente: elaboración de los autores

La Jerarquía de Drenaje fue el parámetro principal, ya que ayuda a comprender en primera instancia la interacción entre el sistema fluvial y las demás características ambientales del territorio de la cuenca, tales como la geología y estructura geológica, el relieve y pendiente, suelos dominantes e impermeabilidad. El análisis cuantitativo propone un esquema de ordenamiento para la red de drenaje que se relaciona con la estructura de bifurcación y su distribución espacial (Figura 6). 
Figura 6. Jerarquía de Drenaje según la metodología de Strahler

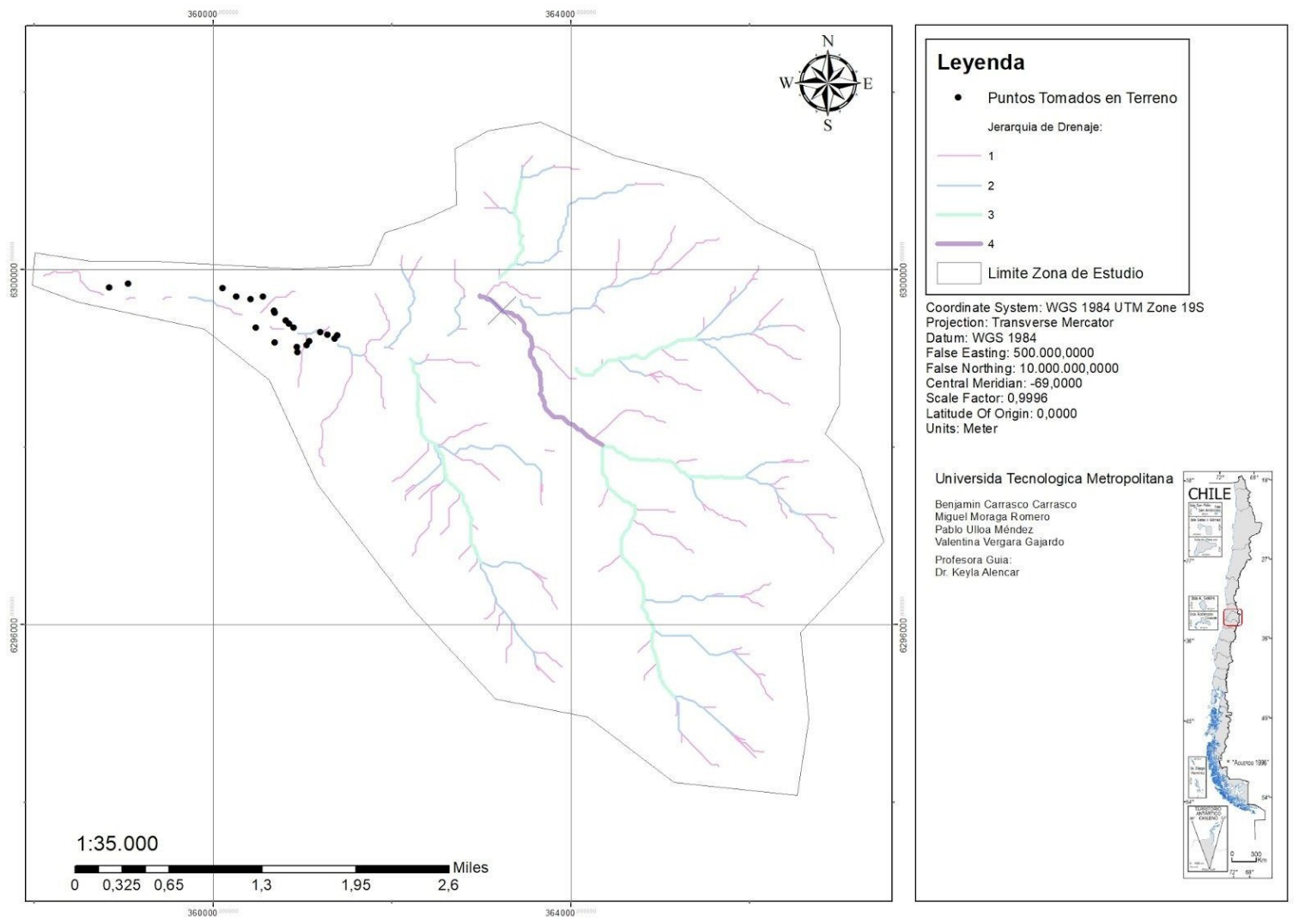

Fuente: elaboración de los autores

\section{CONCLUSIONES}

Los resultados obtenidos a partir de la evaluación de la climatología, morfometría, hidrología y topografía de la microcuenta apunta a la susceptibilidad de ocurrencia de procesos de remoción en masa, inundaciones y aluviones, basándose en el tiempo de concentración (aproximadamente 36.87 minutos), velocidad media de flujo (aproximadamente $15.1 \mathrm{~km} / \mathrm{h}$ ) donde a menores tiempo de concentración de la escorrentía superficial, se conjuga con una mayor susceptibilidad de experimentar crecidas, una vez que la climatología dela zona, la pendiente pronunciadas y la breve duración crean una situación propicia para un desastre natural, en donde los flujos de detritos confluyen al cauce de la quebrada principal y escurren por éste alcanzando las zonas urbanas ubicadas en el abanico aluvial, como por ejemplo el flujo ocurrido en año 1993.

Se destaca el valor de pendiente medio de la microcuenca, dado que el sector estudiado es montañoso, en la en los sectores más altos existen mayores procesos erosivos, mientras que los sectores más bajos las zonas bajas de la cuenca es un área depositacional (abanico aluvial). Se observa el riesgo de remociones en masa superficiales, principalmente en las laderas de mayor pendiente, y en las zonas más cercanas a las cimas, en las laderas colindantes a las principales corrientes de las Quebrada La Pichoca, y al curso superior de la Quebrada Quillayes y Los Maquis, se observa peligro de caídas de roca en bloque con alta peligrosidad, así también en la zona de intersección de las Quebradas Quillayes y San Ramón. Unas pocas pendientes presentan peligro de generar deslizamientos profundos de suelo, y están distribuidas principalmente entre las quebradas Los Maquis, La Pichoca y San Ramón en su curso superior.

En base a los datos obtenidos se sugiere implementar, planos de regulación para la construcción de viviendas aledañas a la zona, planes de seguridad comunitaria para los riesgos vinculados a esta dinámica (consejos comunales, conversaciones de técnicos de riesgo, etc), para que sean expuestas las 
condiciones de vulnerabilidad extrema que presenta la zona, y que hacer frente a los peligros latentes de su entorno, esto con el fin de poder entregar herramientas y los conocimientos necesarios para poder ejercer de manera óptima antes, durante y después de algún suceso adverso sabiendo cómo resguardar la integridad física de la comunidad que habita en esa zona.

\section{REFERÊNCIAS}

ACUÑA FUENTES, Mario Andrés et al. Serie estudio de caso Aluvión de La Florida ocurrido el 3 de mayo de 1993, incluye resumen general. 1995.

CONTRERAS VARGAS, María Teresa et al. Modeling of flash floods in the Andean foothills: reducing the uncertainty associated with the sediment concentrations. 2016.

GIRONÁS, Jorge; SANDOVAL, Jorge. Riesgo de origen hidrometeorológico en la ciudad de Santiago. 2016.

HORTON, Robert E. Erosional development of streams and their drainage basins; hydrophysical approach to quantitative morphology. Geological society of America bulletin, v. 56, n. 3, p. 275-370, 1945.

IBÁÑEZ, S.; MORENO, H.; GISBERT, J. M. I. B. Morfología de las cuencas hidrográficas. Universidad politécnica de Valencia, 2011.

LARA, M. Evaluación del peligro de remociones en masa en Quebrada San Ramón, Santiago Oriente. Santiago, v. 22, p. S3_008, 2009.

MÉNDEZ, Williams et al. Caracterización hidroclimatológica y morfométrica de la cuenca del río San Julián (estado Vargas, Venezuela): aportes para la evaluación de la amenaza hidrogeomorfológica. Cuadernos de Geografia: Revista Colombiana de Geografía, v. 24, n. 2, p. 133-156, 2015.

NARANJO, Jose A.; VARELA, Juan. Flujos de detritos y barro que afectaron el sector oriente de Santiago, el 3 de mayo de 1993. Servicio Nacional de Geología y Minería, 1996.

RÍOS BRIONES, Verónica Pilar. Uso de un modelo lluvia escorrentía para la caracterización de crecidas rápidas en la precordillera andina. El caso de la Quebrada Ramón. 2015. Dissertação de Mestrado. Santiago/Pontificia Universidad Católica de Chile/2015.

RUGIERO, Vanessa; WYNDHAM, Katherine. Identificación de capacidades para la reducción de riesgo de desastre: enfoque territorial de la participación ciudadana en la precordillera de comuna de La Florida, Santiago de Chile. Investigaciones Geográficas, n. 46, p. ág. 57-78, 2013.

SERVICIO NACIONAL DE GEOLOGÍA Y MINERÍA (SERNAGEOMIN). Catastro de los principales desastres naturales por peligros geológicos en Chile y efectos sobre la población y bienes públicos y privados entre 1980 y 2015. Ministerio de Minería, Gobierno de Chile. 2015.

STRAHLER, Arthur N. Part II. Quantitative geomorphology of drainage basins and channel networks. Handbook of Applied Hydrology: McGraw-Hill, New York, p. 4-39, 1964.

VARGAS, Ximena. Corrientes de detritos en la Quebrada de Macul, Chile. Estudio de caudales máximos. Ingeniería del agua, 1999, vol. 6, núm. 4, 1999. 